\title{
A System Approach to Integrated Power Amplifier Analysis and Design
}

\author{
Mihai Banu and Vladimir Prodanov \\ MHI Consulting LLC, New Providence New Jersey, 07974, USA \\ mihaibanu@ieee.org
}

\begin{abstract}
Numerous Power Amplifier circuits are described in the literature, which seem to follow many distinct design approaches. While circuit implementations may be quite different indeed, the underlining system-level signaling inside these circuits follows similar conditions dictated by the common design objective for high efficiency. This paper presents a general theory for analyzing and designing power amplifiers, providing an insight on the fundamental factors limiting the performance, irrespective of the implementation circuits or technology.
\end{abstract}

\section{INTRODUCTION}

Electrical power amplification [1-3] has played a key function in electronic systems ever since Lee de Forest invented the vacuum tube triode in 1906 and Tellegen introduced the pentode tube in 1926. Soon after, electrical engineers worked out the details of designing good class A. B. C Power Amplifiers (PAs). By the second part of the $20^{\text {th }}$ century, this art became a mature and narrow EE specialty mastered by a few, which seemed to have little room left for major innovations. However, the late century market explosion of mobile digital communication devices, such as cellular phones and wireless LANs and the massive introduction of IC technology in everyday life have changed the electronic landscape dramatically, opening a new demand for developing appropriate PAs. The new generation of PA designers much larger in number than before had to adapt the old techniques to the limitations and benefits of using integrated designs. This factor and the wholesale relearning of the old techniques have created a true sense of "black art" in PA design.

In this paper, we show that all basic PAs, irrespective of

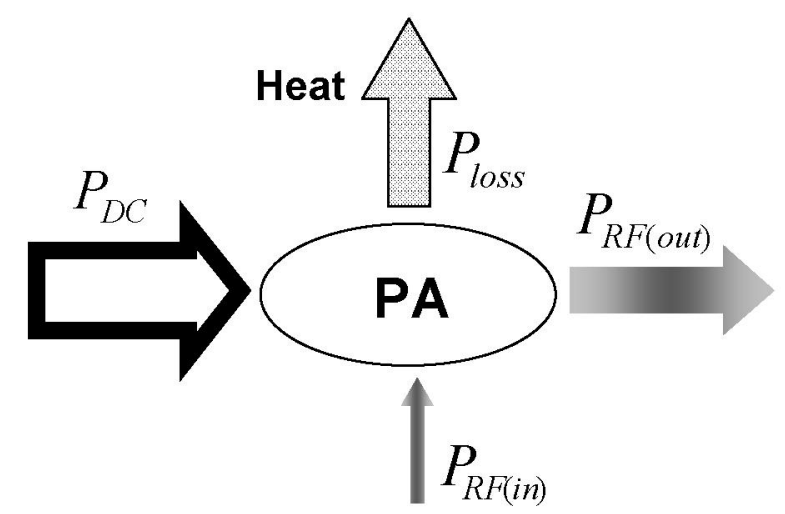

Figure 1: Energy conversion in a PA circuit topologies and implementation technologies, follow certain system-level rules, i.e. rules regarding the internal voltage and current signals, dictated by the overall requirement for efficient energy conversion.

\section{What is A "Power AMPlifier" Circuit?}

The key to understanding PAs is the essential observation that these circuits perform energy (power) conversion, NOT power amplification. Rather than violate the first law of thermodynamics as their name might suggest (energy cannot be amplified!), PAs transfer power from DC to RF. The most important task they have is to do this process efficiently. Figure 1 shows the power balance diagram in a PA. Most power flowing into the circuit comes through the DC lines (input RF power is usually very small in comparison) and power flowing out is divided between desired output RF power and undesired heat loss. The efficiency is high if the loss is small and vice versa.

A typical PA core circuit is shown in Figure 2. A striking fact to the uninitiated is that, in general the PA is a highly nonlinear circuit despite its almost linear input/output behavior. It is quite common for the internal voltages and currents to contain large harmonics. Traditionally, the presence of these harmonics is viewed as a byproduct of the various techniques developed over the years for obtaining high efficiency. This is true for classical current-mode designs such as class AB PAs and modern switched-mode designs. Shortly, it will be shown that internal harmonics are necessary for high efficiency and the only way to minimize loss is by nonlinear effects.

\section{PA INTERNAL SignaLS}

Classical current-mode PAs (Figure 2) operate in class A, $\mathrm{AB}, \mathrm{B}$, or $\mathrm{C}$. For all cases, the transistor drain voltage is sinusoidal, assuming a sinusoidal input. The drain current is sinusoidal only for class A operation, yielding 50\% maximum efficiency. In all other cases, the drain current is limited to a portion of a sinusoidal, yielding higher than $50 \%$ efficiency. Empirically, it seems that the more nonlinear is the drain current the better is the PA efficiency. Next, we show mathematically the validity of this observation.

In steady state, under a sinusoidal excitation of frequency $\omega_{R F}$, the drain voltage and current are periodic functions:

$$
v(t)=V_{D C}+V_{1} \cos \left(\omega_{R F} t+\varphi_{V 1}\right)+\sum_{k=2}^{\infty} V_{k} \cos \left(k \omega_{R F} t+\varphi_{V k}\right)
$$




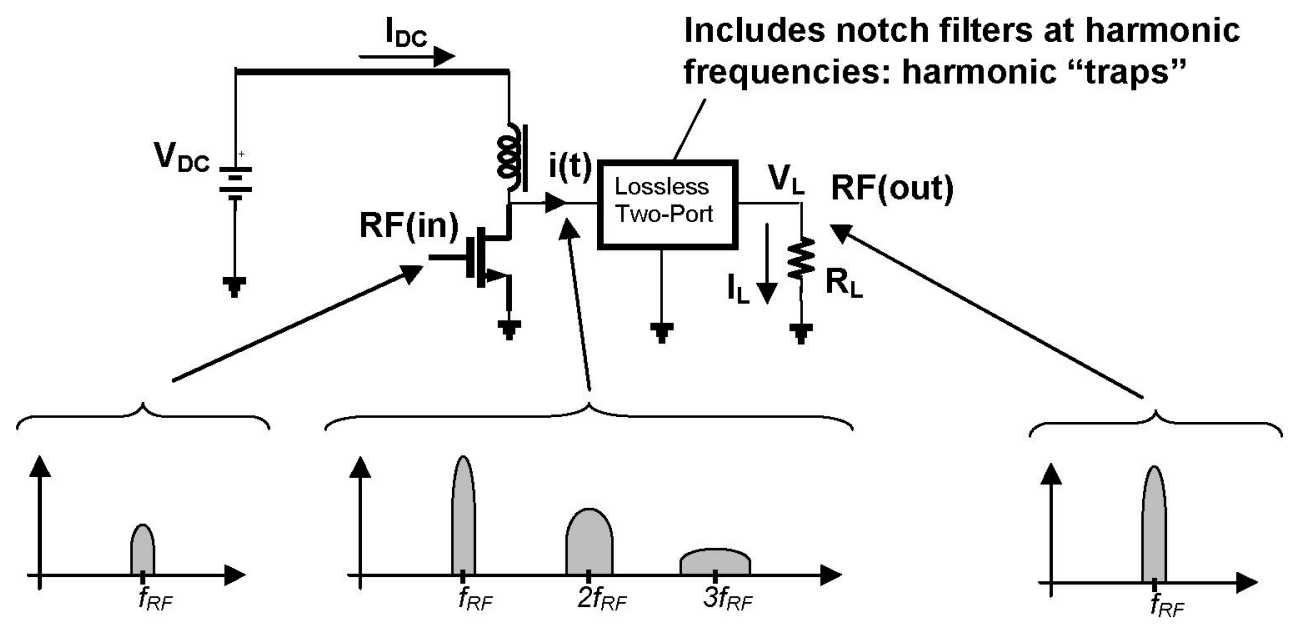

Figure 2: A non-linear PA circuit with linear RF-in/RF-out characteristic

$$
i(t)=I_{D C}+I_{1} \cos \left(\omega_{R F} t+\varphi_{I 1}\right)+\sum_{k=2}^{\infty} I_{k} \cos \left(k \omega_{R F} t+\varphi_{I k}\right)(\mathrm{lb})
$$

where $V_{k}$ and $I_{k}$ are the amplitudes and $\varphi_{V K}$ and $\varphi_{I K}$ are the phases of respective harmonics. The total loss at the drain is calculated by multiplying relations (1a) and (1b) and integrating over a period.. Since all orthogonal products (i.e., voltage and current harmonics having different frequencies) integrate to zero, we have:

$$
\begin{aligned}
P_{\text {loss }}=V_{D C} I_{D C}+ & \frac{1}{2} V_{1} I_{1} \cos \left(\varphi_{V 1}-\varphi_{I 1}\right)+ \\
& \frac{1}{2} \sum_{k=2}^{\infty} V_{k} I_{k} \cos \left(\varphi_{V k}-\varphi_{I k}\right)
\end{aligned}
$$

Relation (2) gives important insights on how the PA converts energy from DC to RF. The total loss $P_{\text {loss }}$ must be a positive quantity since the transistor considered as operating with full voltages and currents is a passive device (transistors do not generate power!), unlike its customary model used for small-signal analysis. Furthermore, the transistor physics forces the DC drain current $I_{D C}$ as defined in Figure 2 to be always positive, which makes the first term in the right hand side of (2) positive. This term is clearly identified as the power delivered into the PA by the DC power supply. The energy conservation law tells us that $P_{\text {loss }}$ must be smaller than the DC power flowing into the PA therefore the second and third terms in the right hand side of (2) must add to a negative number. We can interpret the right hand side of (2) as the superposition of the DC power flowing into the transistor from the DC power supply and a portion of it flowing out of the transistor at RF fundamental and harmonics. Since the biasing choke blocks the RF fundamental and harmonics, the only place the outgoing power can go is the PA load resistor through the lossless twoport. Thus, the PA accomplishes energy conversion: it extracts power at DC from the power supply and delivers a portion of it to the load at RF fundamental and harmonics. Next, we analyze the possibilities to make this process power efficient, i.e., with as small $P_{\text {loss }}$ as possible.

The second term in the right hand side of (2) is essential to the very function of the PA since it represents the fundamental RF power to be delivered to the PA load. This term should be negative with as large as possible magnitude. A necessary condition for this objective is to create fundamental voltage and current signals swinging in opposite directions $\left(180^{\circ}\right.$ phase shift) to make the cosine factor equal to -1 . This is automatically accomplished if the transistor pushes current into a real impedance. Therefore, the two-port lossless network terminated by the PA load resistor must be designed to have a real input impedance at the fundamental frequency. An equivalent way to state this is that the two-port lossless network terminated in the PA load resistor is a filter with a pass-band at the fundamental RF frequency. Naturally, the transistor parasitic capacitances must be included in the network.

The generation of harmonic power represented by the last summing term in the right hand side of (4) must be eliminated for the following reasons. As discussed above, any negative components in the sum would represent respective harmonic power flowing out of the transistor only to be dissipated in the PA load. This is not allowed by the PA linearity requirements. On the other hand, any positive components in the sum would be dissipated in the transistor to the detriment of power efficiency. The only alternative left is to make the harmonic power summation zero.

\section{Zero Harmonic Power AND FDC Ratios}

The last sum in (2) becomes zero when at least one of the voltage or current harmonic magnitudes $\left(V_{k}\right.$ or $\left.I_{k}\right)$ is zero at any particular harmonic frequency. For example, all current-mode PAs (classes A, AB, B, C) have zero voltage harmonics. Likewise, most switched-mode PAs (e.g., class, D, F) use odd harmonic internal voltage and even harmonic internal current. 
Class E PAs realize zero harmonic power through quadrature voltage and current harmonics.

For zero harmonic power and maximum fundamental power, we calculate the power efficiency from relation (2) with the last term eliminated:

$$
P E=\frac{1}{2} \frac{V_{1}}{V_{D C}} \frac{I_{1}}{I_{D C}}
$$

The overall PA efficiency is the product of two "fundamentalcomponent to DC-component" (FDC) signal ratios. This is explicit evidence that the PA efficiency is directly linked to its internal signal harmonics, whose presence in proper amount and phasing can increase the FDC ratios. Figure 3 illustrates the synthesis of elementary functions with even, odd, or all harmonic structures, which have increasing FDC ratios as their

(a)

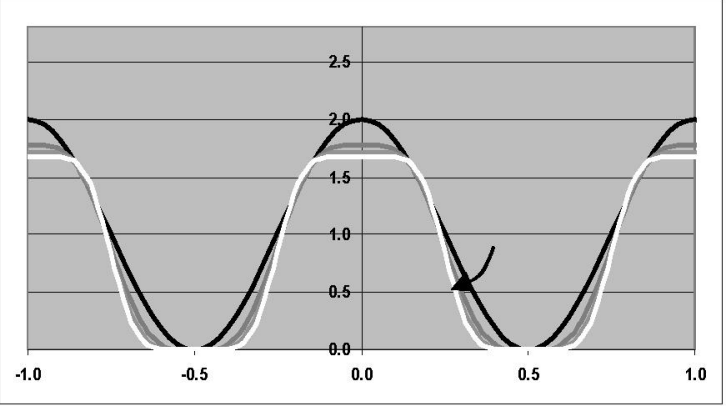

(b)

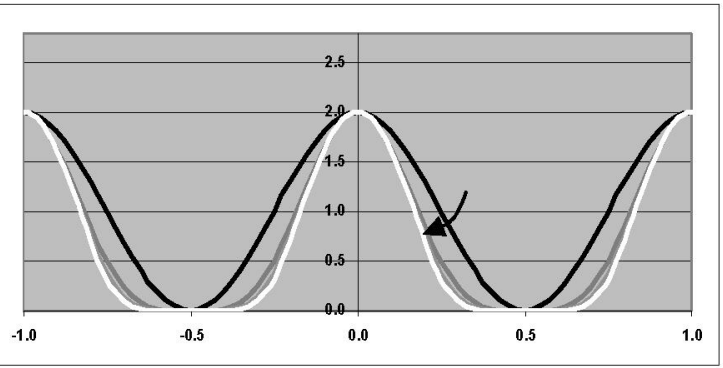

(c)

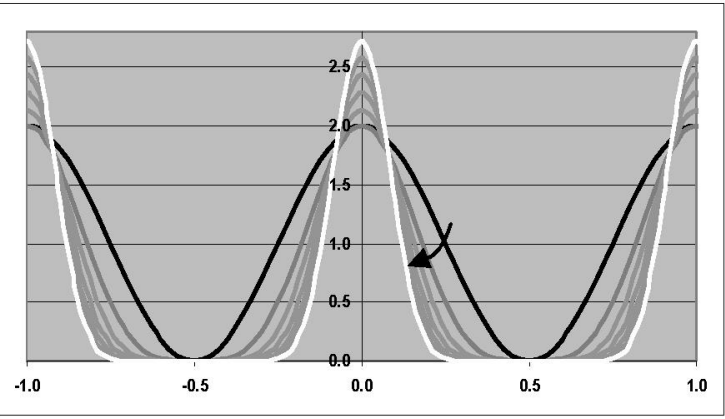

Figure 3: Series of functions with unity fundamental and increasing number of harmonics realizing increasing FDC ratios (a) odd-harmonic functions converging to a square wave (b) even-harmonic functions converging to a half-wave rectified sinusoidal (c) all-harmonic functions converging to an impulse number of harmonics increases. In the limit, the maximum FDC ratios possible are $4 / \pi$ for a square wave, $\pi / 2$ for a halfwave rectified sinusoidal, and 2 for an ideal impulse. Next, we show how by combining these elementary function, we derive the system-level behavior of all known PA structures.

\section{PA EEFFICIENCY TABLES}

Table I compiles the maximum theoretical efficiencies for all possible combinations of internal PA voltage and current signals, which are either square waves, or half-wave rectified sinusoidals, or impulse functions (all are maximum FDC ratio signals) such that the zero harmonic power condition is met and the fundamentals are out of phase. Table II shows the corresponding PA operating classes.

\begin{tabular}{|c|c|c|c|c|c|}
\hline \multirow{2}{*}{\multicolumn{2}{|c|}{ Harmonics }} & \multicolumn{4}{|c|}{ VOLTAGE } \\
\hline & & none & odd & even & all \\
\hline \multirow{4}{*}{$\begin{array}{l}C \\
U \\
R \\
R \\
E \\
N \\
T\end{array}$} & none & $\begin{array}{c}50.0 \\
\%\end{array}$ & $\begin{array}{c}63.6 \\
\%\end{array}$ & $\begin{array}{c}78.5 \\
\%\end{array}$ & $\begin{array}{c}100 \\
\%\end{array}$ \\
\hline & odd & $\begin{array}{c}63.6 \\
\%\end{array}$ & & $\begin{array}{c}100 \\
\%\end{array}$ & \\
\hline & even & $\begin{array}{c}78.5 \\
\%\end{array}$ & $\begin{array}{c}100 \\
\%\end{array}$ & & \\
\hline & all & $\begin{array}{c}100 \\
\%\end{array}$ & & & $\begin{array}{c}100 \\
\%\end{array}$ \\
\hline
\end{tabular}

Table I: Maximum theoretical PA efficiency for different waveform pairing

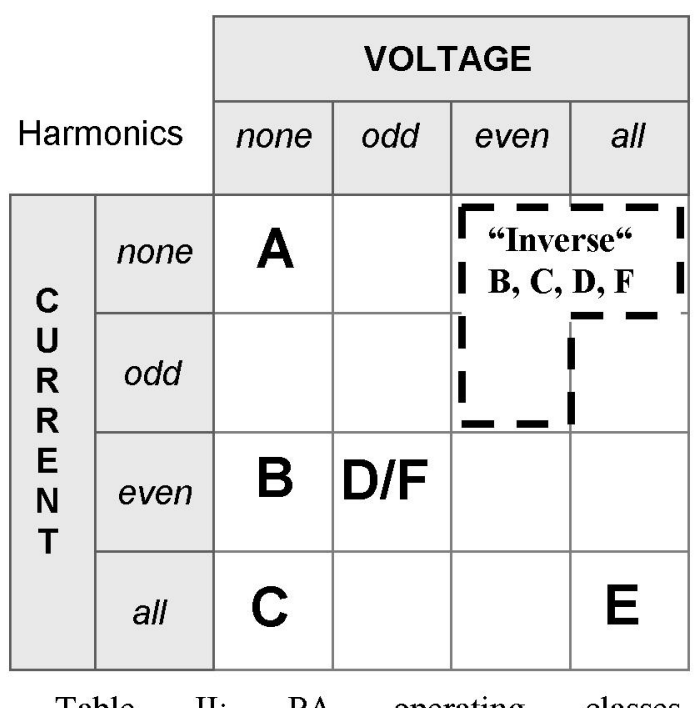

Table II: PA operating classes corresponding to waveform pairing in Table 
The signals in Table I are paired such that either $V_{k}$ or $I_{k}$ is zero except for the pairing for class E operation, when $V_{k}$ and $I_{k}$ are assumed in quadrature (hence the well-known difficulty of designing a good class E PA). In class A operation there are no harmonics, corresponding to the traditional linear amplifier.

\section{FINITE BANDWIDTH CONDITIONS}

The eficiencies in Table I are computed based on ideal functions with infinite number of harmonics. Naturally, within any real PA the voltage and current signals have limited bandwidths. It is instructive to calculate the theoretical PA performance under finite bandwidth conditions, which provide a better upper bound for the expected perfromance in practice. To this end, one can still use relation 3 with appropriate values for the FDC ratios. The latter were calculated with the program Maple for the functions in Figure 3 having a limited number of harmonics. The resulting efficiencies are shown in Table III and Table IV. Simillar considerations are given in [5] and [6].

Table III shows the PA cases where the internal voltage and current have either even harmonics or odd harmonics, but not at the same time such as to guarantee zero harmonic power. Each row corresponds to a maximum number of odd harmonics used and each column corresponds to a maximum number of even harmonics used. Both possibilities, i.e., odd-voltage/evencurrent or odd-current/even-voltage are valid. Table IV shows the PA cases when one of the internal signals (voltage or

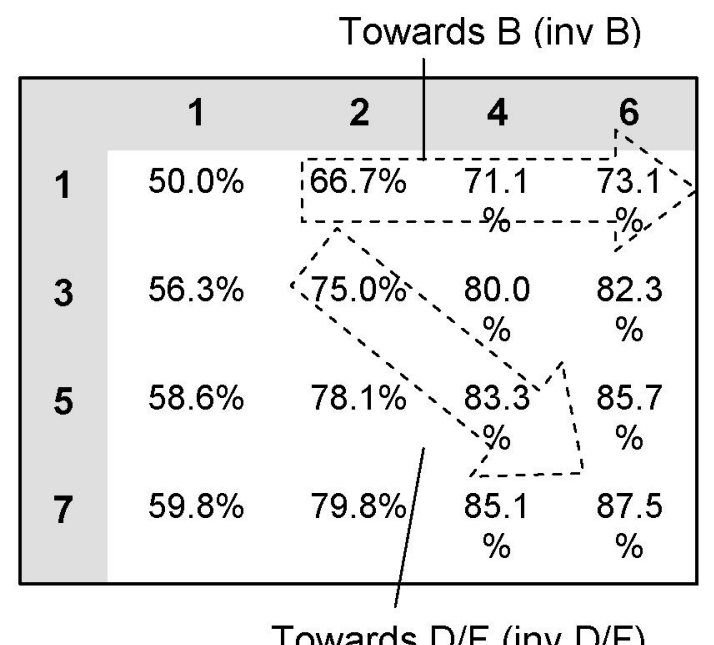

Towards D/F (inv D/F)

Table III: Efficiency for a PA with even harmonics in one internals signal and odd harmonics in the other

\begin{tabular}{|c|c|c|c|c|c|c|c|}
\hline \# Harmonic & $\mathbf{1}$ & $\mathbf{2}$ & $\mathbf{3}$ & $\mathbf{4}$ & $\mathbf{5}$ & $\mathbf{6}$ & $\mathbf{7}$ \\
\hline Efficiency (\%) & 50.0 & 66.7 & 75.0 & 80.0 & 83.3 & 85.7 & 87.5 \\
\hline
\end{tabular}

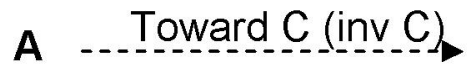

Table IV: Efficiency for a current-mode PA using all harmonics in one of the internal signals (voltage or current) current) has only a fundamental and no harmonics and the other has both even and odd harmonics.

If we consider the rows in Table III as giving the maximum internal voltage harmonics and the culumns as giving the maximum internal current harmonics, the first row demonstrates how the classical class A PA evolves towards a class B PA by the addition of current even harmonics. A comparison with the ideal case of Table 1 shows that using only the second and the forth current harmonics brings the PA efficiency to $71.1 \%$, which is close to the $78.5 \%$, efficiency of the ideal class B. This helps explain the historical success of current-mode PAs.

The diagonal of Table III demonstrates what happens in practical class D/F operation. While these PAs promise $100 \%$ efficiency theoretically, in reality even after using a seventh harmonic in voltage and a sixth harmonic in current, the efficiency reaches only $87.5 \%$, which is respectable but still some distance away from the ideal. This helps explain why audio class D PAs have been succefully developed but RF implementations are extremely challenging. Interestignly, the evolution of class-A into class- $C$ as seen in Table IV yields the same efficiency for the same number of current harmonics with no voltage harmonics.

\section{CONCLUSIONS}

Approaching the PA analysis and design from a high-level system point of view and focusing on the PA DC-to-RF energy conversion process have important benefits. First, it is possible to develop a simple unified theory for the proper understanding of most circuits in this class, irrespective of the implementation technology. Second, a fundamental tradeoff between efficiency and internal circuit bandwidth is substantiated. This is particularly important to set realistic expectations for the practical performance, given a particular implementation technology and operating frequency.

\section{REFERENCES}

[1] S. C. Cripps, RF Power Amplifiers for Wireless Communication, Boston: Artech House, 1999

[2] F. H. Raab, P. Asbeck, S. Cripps, P. B. Kenington, Z. B. Popovic', N Pothecary, J. F. Sevic, and N. O. Sokal, "Power Amplifiers and Transmitters for RF and Microwave," IEEE Trans. Microwave Theory and Techniques, Vol. 50, No. 3, pp. 814-825, March 2002

[3] S. C. Crips, Advanced Techniques in RF Power Amplifier Design, Boston: Artech House, 2002

[4] V. Prodanov and M. Banu, "Power Amplifier Principles and Modern Design Techniques" chapter in "Emerging Wireless Technologies: Circuits, Systems, and Devices "Taylor \& Francis 2007, ISBN: 0849379962

[5] F. H. Raab, "Class-F Power Amplifiers with Maximally Flat Waveforms," IEEE Trans. Microwave Theory and Techniques, Vol. 45, No. 11, pp. 2007-2012, Nov. 1997.

[6] F. H. Raab, "Class-E, Class-C, and Class-F Power Amplifiers Based Upon a Finite Number of Harmonics," IEEE Trans. Microwave Theory and Techniques, Vol. 49, No. 8, pp. 1462 - 1468, August. 2001. 